\section{Mutual benefits?}

A new report in Applied and Environmental Microbiology describes the first identification of a symbiotic bacterium with the ability to enter and reside in mitochondria. Representing a novel clade of $\alpha$-proteobacteria, the bacterium designated as IricES1 — is found in the medically important tick Ixodes ricinus.

Using electron microscopy and in situ hybridization, Beninati et al. report that IricES1 is restricted to ovarian cells of I. ricinus. Interestingly, the authors found that the bacteria were widespread in I. ricinus females from various parts of Europe $-96 \%$ of female ticks were found to be infected; however, IricES1 was absent from males.

On the basis of the very high prevalence of IricES1 in female ticks, its distribution throughout Europe and the absence of sex ratio biases, the authors argue against a parasitic or commensal relationship between IricES1 and I. ricinus, despite the apparent damage the bacteria do to a large number of mitochondria. Instead, they postulate that IricES1 has an obligate mutualistic

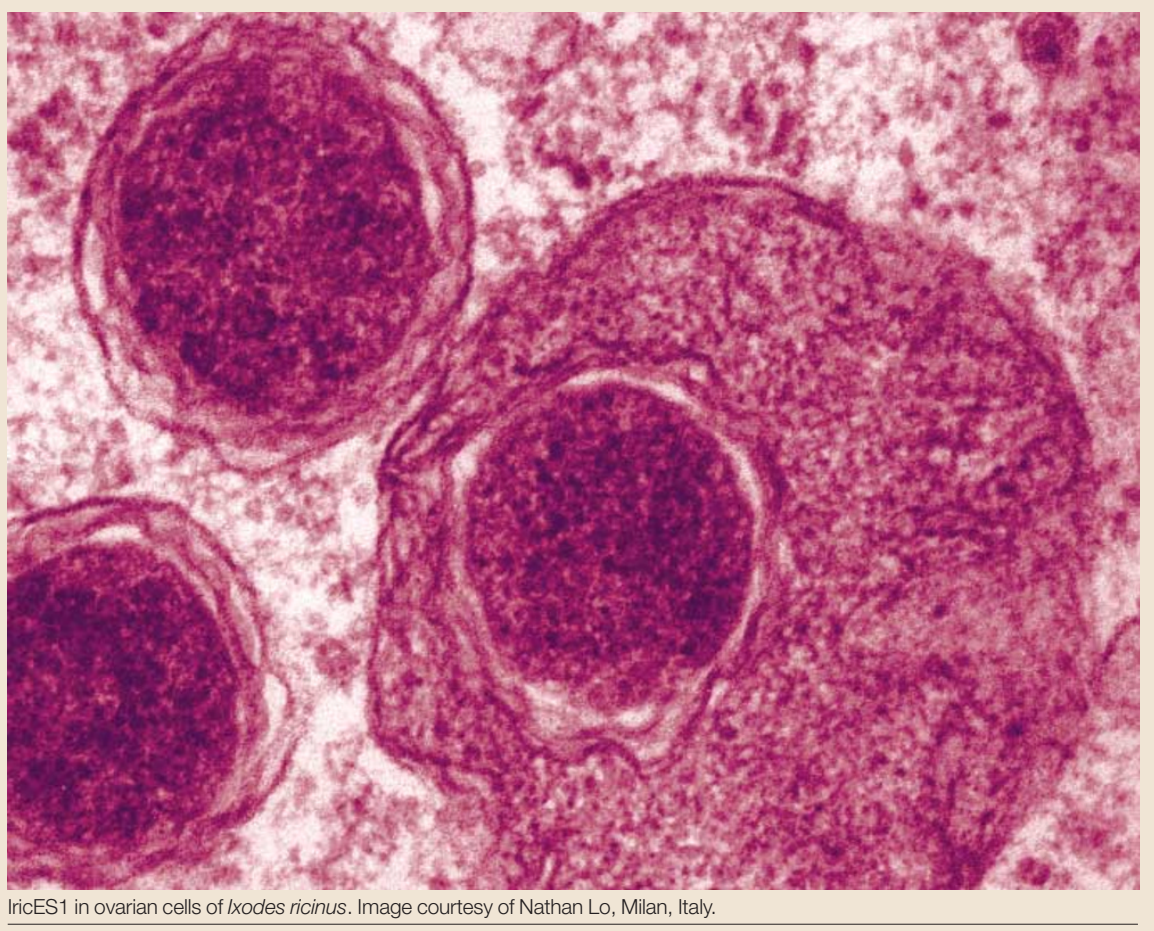

relationship with I. ricinus females.

As IricES1 is only located in the ovarian tissue, it is unlikely to have an important role in host metabolism; however, in a similar manner to Wolbachia in populations of Asobara tabida, IricES1 could be required for I. ricinus oogenesis.

Further studies are required to confirm this hypothesis; however, establishment of an obligate relationship between IricES1 and I. ricinus might provide a model for an unusual type of symbiosis.

Jane Saunders

\section{(4) References and links}

ORIGINAL RESEARCH PAPER Beninati, T. et al. A novel $\alpha$-proteobacterium resides in the mitochondria of ovarian cells of the tick /xodes ricinus. Appl. Environ. Microbiol. May 2004 (doi:10.1128/AEM.70.5.000-000.2004)

\section{ENVIRONMENTAL MICROBIOLOGY}

\section{Suck it up!}

Endophytic bacteria modified by natural gene transfer can improve the phytoremediation of volatile organic compounds such as toluene, according to a recent report in Nature Biotechnology.

Phytoremediation - the use of plants to remove harmful contaminants from the environment - is an emerging bioremediation strategy. Genetic engineering techniques can be used to introduce phytoremediation properties, often from bacteria, into suitable plant species. Another approach, now described by Barac et al., is to introduce the appropriate degradation properties into endophytic bacteria, which are found in the vascular tissues of most plants.

For this proof-of-concept study, Barac et al. used the yellow lupin and Burkholderia cepacia. B. cepacia strain G4 - a soil microorganism that carries a plasmid encoding the toluene catabolic pathway — was conjugated with B. cepacia BU0072, a derivative of the natural endophytic strain B. cepacia L.S.2.4. The resulting toluene-degrading endophytic strain was named B. cepacia VM1330.

Lupins were then inoculated with BU0072, VM1330 or G4 and grown for 21 days. Analysis of the bacterial content of the roots and shoots confirmed that colonization with all three $B$. cepacia species was successful. The effects of the presence of toluene on plant growth were then assessed. In hydroponic culture - soilfree growth in water containing dissolved nutrients — the growth of plants inoculated with VM1330 was unaffected at toluene concentrations up to $1 \mathrm{~g} \mathrm{l}^{-1}$; by contrast, toluene levels $>100 \mathrm{mg} \mathrm{l}^{-1}$ proved to be toxic for the uninoculated controls and plants inoculated with G4 or BU0072. In plants grown in nonsterile soil in a greenhouse, the protective effects of the toluene-degrading plasmid in VM1330 were evident at toluene concentrations up to $500 \mathrm{mg} \mathrm{l}^{-1}$. It was additionally found that plants inoculated with VM1330 released 50-70\% less toluene by evapotranspiration - the combination of evaporation and transpiration - than those inoculated with the control strains, indicating that inoculation with VM1330 improves the degradation of toluene.

B. cepacia expressing the toluene catabolic plasmid therefore not only protects yellow lupins against the toxic effects of toluene but also improves the in planta degradation of this common pollutant. Barac et al. now intend to extend their study to poplar, a deep-rooting tree species used in phytoremediation, and have already identified 150 different poplar endophytes.

Sheilagh Clarkson

\section{(2) References and links}

ORIGINAL RESEARCH PAPER Barac, T. et al. Engineered endophytic bacteria improve phytoremediation of water-soluble, volatile, organic pollutants. Nature Biotechnol. 22, 583-588 (2004) 